\title{
Prediction of treatment responses to neoadjuvant chemotherapy in breast cancer using contrast-enhanced ultrasound
}

\author{
Yunxia Huang ${ }^{1,2}$, Jian Le ${ }^{1,2}$, Aiyu Miao ${ }^{1,2}$, Wenxiang Zhi ${ }^{1,2}$, Fen Wang ${ }^{1,2}$, Yaling Chen ${ }^{1,2}$, Shichong Zhou ${ }^{1,2}$, \\ Cai Chang ${ }^{1,2}$ \\ ${ }^{1}$ Department of Ultrasonography, Fudan University Shanghai Cancer Center, Shanghai, China; ${ }^{2}$ Department of Oncology, Shanghai Medical \\ College, Fudan University, Shanghai, China \\ Contributions: (I) Study conception and design: C Chang, S Zhou; (II) Administrative support: Y Chen, F Wang, W Zhi, A Miao; (III) Provision \\ of study materials or patient recruitment: W Zhi, J Le; (IV) Data collection: Y Huang; (V) Data analysis and interpretation: Y Huang, S Zhou; \\ (VI) Manuscript writing: All authors; (VII) Final manuscript approval: All authors. \\ Correspondence to: Shichong Zhou; Cai Chang. Department of Ultrasonography, Fudan University Shanghai Cancer Center, Department of Oncology, \\ Shanghai Medical College, Fudan University, No 270, Dong'an Road, Xuhui District, Shanghai 200032, China. \\ Email: sczhou@hotmail.com; changcai_fuscc@hotmail.com.
}

\begin{abstract}
Background Elucidation the efficacy of neoadjuvant chemotherapy (NAC) in breast cancer is important for informing therapeutic decisions. This study aimed at evaluating the potential value of contrast-enhanced ultrasound (CEUS) parameters in predicting breast cancer responses to NAC.

Methods: We performed CEUS examinations before and after two cycles of NAC. Quantitative CEUS parameters [maximum intensity (IMAX), rise time (RT), time to peak (TTP), and mean transit time (mTT)], tumor diameter, and their changes were measured and compared to histopathological responses, according to the Miller-Payne Grading (MPG) system (score 1, 2, or 3: minor response; score 4 or 5: good response). Prediction models for good response were developed by multiple logistic regression analysis and internally validated through bootstrap analysis. The receiver operating characteristic (ROC) curve was used to evaluate the performance of prediction models.
\end{abstract}

Results: A total of 143 patients were enrolled in this study among whom 98 (68.5\%) achieved a good response and while 45 (31.5\%) exhibited a minor response. Several imaging variables including diameter, IMAX, changes in diameter ( $\triangle$ diameter), IMAX $(\triangle \mathrm{IMAX})$ and TTP $(\triangle T T P)$ were found to be significantly associated with good therapeutic responses $(\mathrm{P}<0.05)$. The areas under the curve $(\mathrm{AUC})$ increased from 0.748 to 0.841 in the multivariate model that combined CEUS parameters and molecular subtypes with a sensitivity and specificity of $0.786,0.745$, respectively. Tumor molecular subtype was the primary predictor of primary endpoint.

Conclusions: CEUS is a potential tool for predicting responses to NAC in locally advanced breast cancer patients. Compared to the other molecular subtypes, triple negative and HER2+/ER- subtypes are more likely to exhibit a good response to NAC.

Keywords: Breast cancer; neoadjuvant chemotherapy (NAC); contrast-enhanced ultrasound (CEUS); treatment response prediction

Submitted Nov 18, 2020. Accepted for publication Mar 09, 2021.

doi: $10.21037 /$ gs-20-836

View this article at: http://dx.doi.org/10.21037/gs-20-836

\section{Introduction}

Neoadjuvant chemotherapy (NAC) is the standard therapeutic option for locally advanced breast cancer patients. It is used to downsize tumor volumes (1). These therapeutic options enable inoperable patients to be treated with operable ways or operable patients converting from 
mastectomy to breast-conserving therapy. In addition, NAC allows in vivo assessment of tumor responses to therapy and, therefore, chemosensitivity. For example, capecitabine adjuvant therapy has been shown to be efficacious against triple-negative breast cancer in patients with residual cancer following NAC (2). Effective tumor treatment is associated with better recurrence-free survival and overall survival outcomes (3). Due to the different histopathological and molecular cancer types, it is difficult to assess the responses to NAC. For instance, there might be no apparent reduction in mass size, even in positively responding tumors. Sometimes, the therapy might result in tumor fragmentation, which limits tumor mass assessment (4). It has been reported that over $40 \%$ of NAC administered patients exhibit a poor pathological response. In such cases, the whole cycle of neoadjuvant therapy is associated with the delayed initiation of another, more effective treatment. This leads to an unnecessary exposure of the patient to toxic side-effects (e.g., hematological ones) of the drug. Therefore, a reliable prediction model for the responses to NAC will inform the optimal selection of patients for surgical procedures or NAC.

Conventionally, tumor size based preoperative radiological assessment is used to evaluate therapeutic responses to NAC. Magnetic resonance imaging (MRI) is the most accurate medical imaging modality for monitoring the progress of NAC treatment in breast cancer patients. Studies have reported the high interobserver variability of MRI for response patterns and tumor diameter in evaluating NAC responses (5). Moreover, a definite standard for drawing region of interest (ROI) of non-mass enhancement lesion has not been developed. These limitations inhibit extensive clinical applications of MRI during NAC response evaluation. Relatively, MRI is also expensive and is contraindicated to patients with claustrophobia, pacemakers, and metallic objects within the body. Therefore, it is not practical to frequently scan patients on NAC therapy using MRI. Other evaluation methods include clinical examinations, mammography, positron emission tomography/computed tomography (PET/ CT) and ultrasound (US) imaging. Among these techniques, US is affordable, widely available and can be used to accurately assess breast tumor size during NAC treatment (6). Other US image features and techniques associated with NAC response include acoustic shadowing and tumor echogenicity $(7,8)$. Moreover, quantitative US utilizes radiofrequency data to estimate various tissue physical properties (e.g., backscatter coefficient) that are important in response prediction $(9,10)$.
Breast cancer heterogeneity is associated with various biological tumor behaviors as shown by radiological imaging (11). Since the overall tumor shrinkage manifests later than microstructural or physiologic alterations, tumor morphology might not provide strong evidence of the real tumor status during NAC therapy (12). Therefore, tumor angiogenesis is the most commonly evaluated for predicting early responses to NAC (13).

Conventional color Doppler imaging has a high diagnostic value in differentiating between benign and malignant breast lesions. However, Doppler imaging is associated with low sensitivity to small vessels $(<200 \mathrm{~mm})(14)$. When used to examine contrast medium-microbubbles within tumor vessels, contrast-enhanced ultrasound (CEUS) has been shown to be more sensitive (15). It has been reported that CEUS performance when assessing the mass differentiation of breast lesions is as accurate as MRI (16). Furthermore, software-assisted time intensity curves (TIC) are gaining momentum in the assessment of lesion perfusions during CEUS imaging $(17,18)$. It is, therefore, possible to evaluate qualitative as well as quantitative tumor blood flow changes during NAC treatment (19). Blood perfusion changes in CEUS are highly correlated with pathological response efficacies (20-22). So far, studies using CEUS to evaluate of the efficacy of NAC in breast cancer have been limited to small sample sizes (17-92 patients), without incorporating clinic-pathological predictors $(17,20,21,23,24)$.

Therefore, this study aimed at evaluating the correlation between CEUS parameters and NAC responses in breast cancer using a large sample size of breast cancer patients. Then, a model based on predictive CEUS and clinicpathological features was established and its ability to predict responses to NAC in breast cancer patients was validated.

We present the following article in accordance with the STARD reporting checklist (available at http://dx.doi. org/10.21037/gs-20-836).

\section{Methods}

\section{Patients}

This prospective study was approved by the institutional ethical review board of the Fudan University Shanghai Cancer Center (1303119-NSFC). The study was conducted in accordance with the Declaration of Helsinki (as revised in 2013). And written informed consent was obtained from all the study participants. The inclusion criteria were: (I) female 


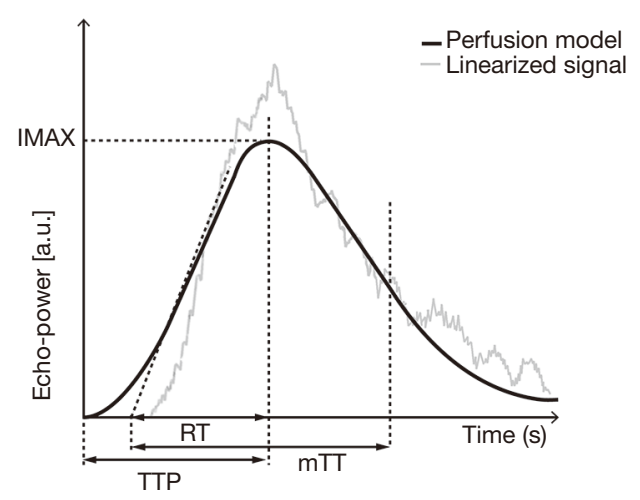

Figure 1 TIC analysis of CEUS. Y-axis of the time-intensity graphs: echo power (arbitrary units, au); x-axis: absolute time seconds (s). TIC, time intensity curves; CEUS, contrast-enhanced ultrasound.

patients with biopsy-proven initial invasive breast cancer; (II) patients with initial clinical stage II or III breast cancer; (III) the patient was previously untreated; and (IV) patients with adequate bone marrow, hepatic, cardiac, as well as renal functions. The exclusion criteria were: (I) patients with initial diagnosis of stage IV breast cancer; (II) presence of malignancy in other organs, regardless of the primary tumor metastasis; and (III) patients subjected to surgery another hospital. A total of 158 patients with biopsy-confirmed invasive breast cancer, who were scheduled to be prescribed with NAC and breast surgery were enrolled in this study. Enrollment was done between August, 2015 and June, 2017. Study participants were subjected to 6 to 8 cycles of NAC therapy according to standard protocols from the National Comprehensive Cancer Network (NCCN) guidelines.

\section{Data collection}

Histological characteristics of the primary tumor [expression of hormone receptors, Human epidermal growth factor receptor-2 (HER2) status, Ki67 value, tumor dimensions and grade, histological tumor type, TMN stage, as well as molecular subtype] and clinical characteristics of each study participant (age at diagnosis) were retrieved from the institutional database.

\section{Sonography and CEUS examination}

Study participants were subjected to US and CEUS at 2 time points: within 1 week before initiation of NAC and after two cycles of therapy (defined as pre- and post- treatment US/CEUS). In case of the presence of multifocal/ multicentric/bilateral lesions, the largest lesion was chosen for estimation. The examinations were finalized using the Esaote MyLab 90 system with a LA532 transducer with a frequency of 4-13 MHz (Esaote, Genova, Italy). At each site, the largest diameter of the tumor was first measured using the two-dimensional US by one researcher (A.Y.M., more than 5 years of experience in breast US) who was blinded to the study. Continuous scanning with color Doppler imaging was then applied through the entire tumor to determine the plane with the highest tumor vascularity, which was marked as the CEUS plane. For CEUS examination, $2.4 \mathrm{~mL}$ sulfur hexafluoride microbubbles (SonoVue, Bracco, Italy) were injected through the antecubital vein using a 20 -gauge intravenous cannula and flushed using $5 \mathrm{~mL}$ of $0.9 \%$ sodium chloride. The US probe and upper bodies of the patients were immobilized to avoid any artifacts in the images. Dynamic imaging was immediately initiated after the injection of the contrast agent and performed for $6 \mathrm{~min}$. The gain and other settings were optimized at the beginning of each examination, and were not changed throughout the procedure.

\section{CEUS analysis}

The obtained dynamic images were saved in the DICOM format and analyzed by the SonoLiver v1.1.15.0 software (TomTec GmbH, Munich, Germany), which automatically drew a time-intensity curve (TIC). An ROI with optimum visualization of the tumor was selected, avoiding areas with ligaments, fascia, calcifications, and necrosis. Among various algorithms $(17,20,21,23-25)$ the following 4 yielded the most promising results and were, therefore, selected and used to calculate the imaging parameters for analysis in this study (Figure 1):

(I) Maximum intensity (IMAX; units, a.u.), the maximal signal intensity of the TIC during tumor perfusion.

(II) Rise time (RT; units, s), time that the curve takes to increase from the starting point to the peak.

(III) Time to peak (TTP; units, s), time from the starting point to the IMAX.

(IV) Mean transit time (mTT; units, s), circulation time of the contrast agent in the area under investigation.

Two researchers (W.X.Z. and F.W. with more than 8 years of experience in interpreting breast CEUS) who were blinded to the study reviewed the CEUS videos. The results 
were measured three times, and the average value calculated. When there was no agreement, a third radiologist (S.C.Z., with 10 years of experience in breast CEUS) reviewed the images to reach a consensus.

\section{Histopatbological examination and assessment of pathological response to treatment}

Pretreatment core biopsies were routinely processed to evaluate the histological and biological characteristics of breast cancer. The expression levels of estrogen receptor (ER) and progesterone receptor (PR) as well as the Ki67 index were expressed as the percentage of cells that stained positively on immunohistochemistry (IHC). HER2 positivity was defined as 3+ using IHC and/or fluorescence in situ hybridization.

Surrogate molecular subtypes were defined based on the 2011 St. Gallen guidelines as follows (26):

* Luminal A (ER and/or PR positive, HER2 negative and Ki67 <14\%);

* Luminal B/HER2- (ER and/or PR positive, HER2 negative and Ki67 $\geq 14 \%$ );

* Luminal B/HER2+ (ER and/or PR positive, HER2 positive);

* HER2+/ER- (ER and PR negative and HER2 positive);

* Triple negative (ER, PR and HER2 negative).

Responses to NAC were assessed using the MillerPayne Grading (MPG) based on cancer cellularity changes between the core pretreatment biopsy and the surgical specimen (27). The measurement scale was: Grade 1, no change or some alterations in individual malignant cells, but no reduction in overall cellularity; Grade 2, a minor loss of tumor cells (up to $30 \%$ ), but the overall cellularity was still high; Grade 3, an estimated 30\% to $90 \%$ reduction in tumor cells; Grade 4, a marked disappearance of tumor cells (>90\%), with only small clusters or widely dispersed individual cells remaining; and Grade 5, no identifiable malignant cells, vascular fibroelastotic stroma often contains macrophages. Residual ductal carcinoma in situ (DCIS) was acceptable. In this study, Grades 1-3 were defined as the minor response group, while Grades 4 and 5 were defined as the good response group.

\section{Statistical analysis}

Clinical data, biological characteristics of the tumor, and imaging-derived features were tested as possible predictors of good response to NAC therapy. Two models were established as follows: in the first model, clinical data, biological tumor characteristics, and US diameter features were included. Univariate analyses were performed using Student's $t$-test, Mann-Whitney U test, and Pearson's $\chi^{2}$ or Fisher's exact test as appropriate. In the univariate analysis, $\mathrm{P}<0.1$ was considered to be statistically significant. The backward logistic regression method was used for the selection of independent variables in logistic regression with entry $\mathrm{P}=0.05$ and removal $\mathrm{P}=0.1$. There were significant differences in the post-treatment parameters. Therefore, we focused on the changes in good and minor responses. Percentage changes in CEUS parameter value (PV), diameter, IMAX, RT, TTP, and mTT ( $\mathrm{PV}_{\text {pre, }} \mathrm{PV}$ at prechemotherapy; $\mathrm{PV}_{\text {poss }}, \mathrm{PV}$ at post-chemotherapy) were calculated using the following formula:

$$
\Delta \mathrm{PV}=\frac{(\mathrm{PV} \text { post }-\mathrm{PV} \text { pre })}{\mathrm{PV} \text { pre }} \times 100 \%
$$

In the second model, CEUS parameters were also evaluated as possible predictors of good response to NAC therapy. Features identified by univariate logistic regression as additional outcome predictors, along with the variables identified in the first model, were included in the model (herein referred to as model 2).

Model performance was evaluated through discrimination and calibration. A receiver operating characteristic (ROC) curve and the area under curve (AUC) were generated to assess the discriminative ability of the prediction model. Predictive performance of the model was validated by bootstrap resampling, which was repeated 1,000 times. Sensitivity and specificity were calculated using the best cut-off score for the clinical prediction rule and the Youden index for the ROC. The Hosmer-Lemeshow test was used for model calibration. To account for missing data $(<5 \%)$, multiple imputation was performed in the multivariate logistic analysis.

Data analysis was performed using SPSS v25.0 (SPSS, Inc., IMB Company Chicago, IL, USA) and the bootstrap techniques in R statistics (V.3.6.2, http://www.r-project.org). Significance levels were set at a two-sided $\mathrm{P}$ value of 0.05 .

\section{Results}

\section{Patient characteristics}

A total of 15 patients were excluded from the study because: 5 patients did not present themselves for a second CEUS 
Table 1 Summary of the clinical parameters of the patients included in the study

\begin{tabular}{|c|c|c|c|c|}
\hline & Total $(n=143)$ & Good-response $(n=98)$ & Minor-response $(n=45)$ & $P$ values \\
\hline (c)T stage & & & & $0.003^{*}$ \\
\hline $\mathrm{T}_{1}$ & $23[16]$ & 19 [20] & $4[9]$ & \\
\hline $\mathrm{T}_{2}$ & $47[34]$ & $31[32]$ & $16[36]$ & \\
\hline (c) N stage & & & & 0.390 \\
\hline $\mathrm{N}_{0}$ & 19 [13] & 14 [14] & 5 [11] & \\
\hline $\mathrm{N}_{1}$ & 39 [28] & 32 [33] & $7[16]$ & \\
\hline $\mathrm{N}_{2}$ & 35 [25] & 20 [21] & 15 [34] & \\
\hline Lumianl A & $20[14]$ & $8[8]$ & $12[27]$ & \\
\hline Lumianl B/HER2- & $35[24]$ & $18[18]$ & $17[37]$ & \\
\hline Lumianl B/HER2+ & 26 [18] & $18[18]$ & $8[18]$ & \\
\hline HER2+/ER- & $33[24]$ & $31[32]$ & $3[7]$ & \\
\hline Triple negative & $28[20]$ & $23[24]$ & $5[11]$ & \\
\hline Histology & & & & $0.001^{*}$ \\
\hline Ductal/other & $126[88]$ & 91 [93] & $35[78]$ & \\
\hline Lobular/mixed & 17 [12] & 7 [7] & 10 [22] & \\
\hline
\end{tabular}

Except for age (range), data are number of patients. Data in parentheses are percentage or range. $P$ values for difference were determined by chi-square test or fisher's exact test for categorical variables. ${ }^{*}$, statistically significant differences $(P<0.1)$. HER2, human epidermal growth factor receptor-2; ER, estrogen receptor.

examination for personal reasons; 6 patients were not subjected to surgical procedures due to metastatic progression while 4 patients did not have their postoperative pathological data. A total of 143 patients were included in this study from among whom, 98 (68.5\%) exhibited a good response, while 45 (31.5\%) exhibited a minor response to NAC. Of these 143 patients, 9 had missing data for one or more potential parameters: 7 for age, 3 for tumor size, and 2 for lymph node status. More than $60 \%$ of the patients were node-positive. $T$ stage, molecular subtype, and histology satisfied the inclusion threshold for the univariate analysis (Table 1).

\section{CEUS and US evaluation of NAC}

At the initial NAC therapy stage, CEUS revealed hyperenhancement in all tumors without significant differences between the two groups $(\mathrm{P}>0.05$, Table 2). After two cycles of treatment, there was a decrease in diameter and IMAX as well as an increase in RT, TTP, and mTT in most patients (Figure 2). The diameter, IMAX, and their changes were significantly different between the good and minor responders $(\mathrm{P}<0.05)$. CEUS parameters RT, mTT, and their changes were not significantly different between the two groups $(\mathrm{P}>0.05)$. Although there were no significant differences in TTP between the two groups, TTP changes were higher in good responders than in minor responders $(\mathrm{P}<0.05)$.

\section{Model establishment and performance}

In the univariate regression analysis, factors with a significant predictive value were molecular subtype, diameter, $\triangle$ diameter, IMAX, $\triangle \mathrm{IMAX}$ and $\triangle T$ TTP. Since relative changes in $\mathrm{PV}$ were calculated based on pre- 
Table 2 CEUS parameters before and after NAC for discriminating between good and minor responders

\begin{tabular}{|c|c|c|c|c|c|c|c|c|c|}
\hline & \multicolumn{3}{|c|}{ Pre-NAC } & \multicolumn{3}{|c|}{ Post-NAC } & \multicolumn{3}{|c|}{ Percent changes } \\
\hline IMAX (a.u.) & $4,608 \pm 13,569$ & $3,947 \pm 3,070$ & 0.235 & $1,297 \pm 2,264$ & $2,913 \pm 4,141$ & $0.015^{*}$ & $-46.44 \pm 34.70$ & $-5.8 \pm 29.00$ & $0.008^{*}$ \\
\hline TTP (s) & $19.34 \pm 2.21$ & $18.53 \pm 3.05$ & 0.325 & $26.54 \pm 5.56$ & $21.83 \pm 4.56$ & 0.223 & $20.36 \pm 35.93$ & $6.70 \pm 9.59$ & $0.026^{*}$ \\
\hline $\mathrm{mTT}(\mathrm{s})$ & $35.96 \pm 43.49$ & $31.96 \pm 11.22$ & 0.712 & $35.79 \pm 30.68$ & $42.24 \pm 28.20$ & 0.643 & $19.15 \pm 54.04$ & $40.32 \pm 38.39$ & 0.657 \\
\hline
\end{tabular}

Values are presented as means \pm SD. $\mathrm{P}$ values for difference were determined by Student's $t$-test or Mann-Whitney U test. Statistically significant differences $(P<0.1)$. CEUS, contrast-enhanced ultrasound; NAC, neoadjuvant chemotherapy; IMAX, maximum intensity; RT, rise time; TTP, time to peak; $\mathrm{mTT}$, mean transit time.
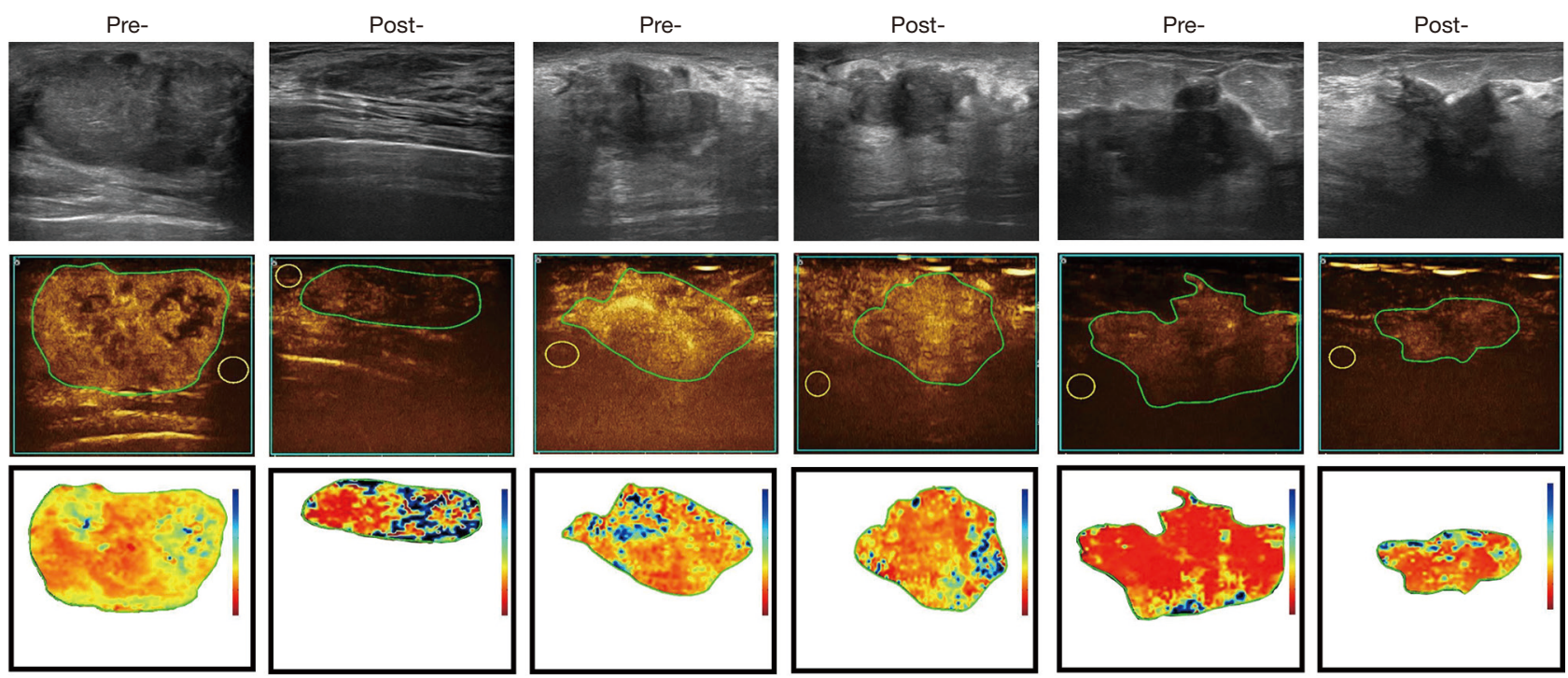

A ร
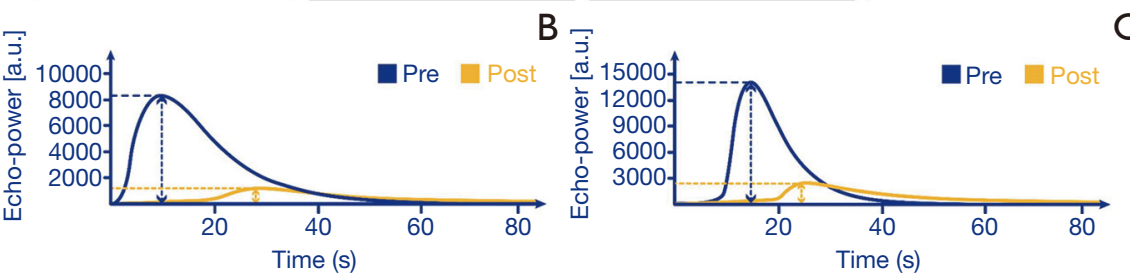

C

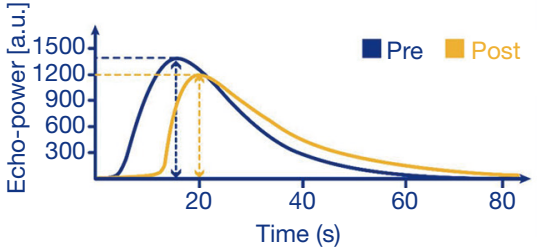

Figure 2 Various CEUS changes in three patients following NAC. At baseline before NAC, conventional US showed hypoechoic, irregularly shaped lesions with indistinct margins (A-C, pre-). After two cycles of treatment (A-C, post-): (A) conventional US showed a reduction in tumor size. CEUS showed reduced diffusion and a prolonged enhancement time in the TIC curve. This patient had triplenegative cancer and was confirmed to have had a good response to treatment. No residual cancer was found in the final pathological analysis. (B) US did not show apparent changes in tumor size. CEUS showed homogeneous isoenhancement with a prolonged enhancement time. The HER2+/ER- patient was confirmed to have had a good response in the final pathological analysis. (C) US showed a reduction in tumor size, while CEUS showed homogeneous hyperenhancement with an increase in IMAX and rapid RT on the TIC curve. This was a luminal B/HER2 - patient who achieved a minor response in the pathological analysis. (below), Color-coded pictures of the quantitative illustrations of these patients are shown in (A-C). CEUS, contrast-enhanced ultrasound; NAC, neoadjuvant chemotherapy; US, ultrasound; TIC, time intensity curves; HER2, human epidermal growth factor receptor-2; ER, estrogen receptor; IMAX, maximum intensity; RT, rise time; pre-, pretreatment; post-, post-treatment. 
Table 3 Results of the logistic regression for predicting good responses to NAC in model 1

\begin{tabular}{lccc}
\hline Characteristic & Odds ratio & $95 \% \mathrm{Cl}$ & P values \\
\hline Diameter & 0.965 & $0.937-0.994$ & 0.017 \\
Breast cancer subtype & & & 0.001 \\
Luminal B/HER2+ & 1 & reference & 0.078 \\
Luminal A & 0.326 & $0.093-1.135$ & 0.090 \\
Luminal B/HER2- & 0.383 & $0.126-1.163$ & 0.124 \\
HER2+/ER- & 2.739 & $0.760-9.874$ & 0.234 \\
Triple-negative & 2.200 & $0.600-8.074$ & 0 \\
\hline
\end{tabular}

NAC, neoadjuvant chemotherapy; Cl, confidence interval; HER2, human epidermal growth factor receptor-2; ER, estrogen receptor.

Table 4 Results of the logistic regression for predicting good responses to NAC in model 2

\begin{tabular}{lccc}
\hline Characteristic & Odds ratio & $95 \% \mathrm{Cl}$ & $\mathrm{P}$ values \\
\hline $\begin{array}{l}\Delta \text { diameter } \\
\text { Breast cancer subtype }\end{array}$ & 0.965 & $0.934-0.997$ & 0.033 \\
Luminal B/HER2+ & 1 & & 0.001 \\
Luminal A & 0.420 & reference & $0.106-1.672$ \\
Luminal B/HER2- & 0.326 & $0.094-1.124$ & 0.076 \\
HER2+/ER- & 3.939 & $0.947-16.372$ & 0.059 \\
Triple-negative & 2.872 & $0.702-11.755$ & 0.234 \\
$\Delta$ IMAX & 0.966 & $0.950-0.982$ & 0.000 \\
$\Delta$ TTP & 1.016 & $1.001-1.030$ & 0.032 \\
\hline
\end{tabular}

NAC, neoadjuvant chemotherapy; HER2, Human epidermal growth factor receptor-2; ER, estrogen receptor; $\triangle I M A X$, percentage changes in maximum intensity; $\triangle \mathrm{TTP}$, percentage changes in time to peak; $\mathrm{Cl}$, confidence interval; HER2, human epidermal growth factor 2.

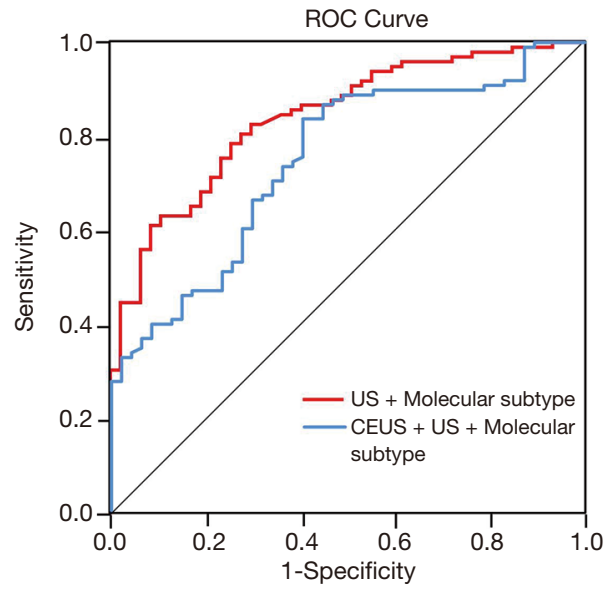

Figure 3 AUCs of possible combinations of imaging parameters and tumor subtype to predict a good response. AUC, areas under the curve. and post-PV, PV (diameter, IMAX) were removed from the model due to possible collinearity issues. Therefore, the model with molecular subtype and $\Delta$ diameter were selected as the model 1 (Table 3), while CEUS parameters ( $\triangle \mathrm{IMAX}$ and $\triangle \mathrm{TTP}$ ) were added to model 2 (Table 4). The AUC for model 2 was higher than model 1 (0.841, 0.748, respectively, $\mathrm{P}=0.001$ ) (Figure 3). The optimism-corrected AUC obtained from bootstrap resampling were 0.748 (model 1) and 0.840 (model 2), suggesting a good internal validation. The best cut-off, sensitivity, specificity for model 1 and model 2 were $0.592,83.7 \%, 59.6 \%$ and $0.658,78.6 \%$, $74.5 \%$, respectively (Table 5). The Hosmer-Lemeshow test was significant $(\mathrm{P}=0.241,0.701$, respectively), indicating that the model had a good fit. These results suggested that a combination of molecular subtype and imaging parameters 
Table 5 AUC-ROC, bootstrap AUC-ROC, and diagnostic accuracy in the multivariable logistic regression of model 1 and model 2

\begin{tabular}{ccccccccc}
\hline & AUC-ROC & $95 \% \mathrm{Cl}$ & Bootstrap AUC-ROC & $95 \% \mathrm{Cl}$ & Optimism & Accuracy & Sensitivity & Specificity \\
\hline Model 1 & 0.748 & $0.665-0.831$ & 0.748 & $0.666-0.830$ & 0.592 & 0.779 & 0.837 & 0.596 \\
Model 2 & 0.841 & $0.776-0.906$ & 0.840 & $0.775-0.905$ & 0.658 & 0.772 & 0.786 & 0.745 \\
\hline
\end{tabular}

Bootstrap repetitions with 1,000 times. AUC, areas under the curve; ROC, receiver operating characteristic curve; $\mathrm{Cl}$, confidence interval.

A

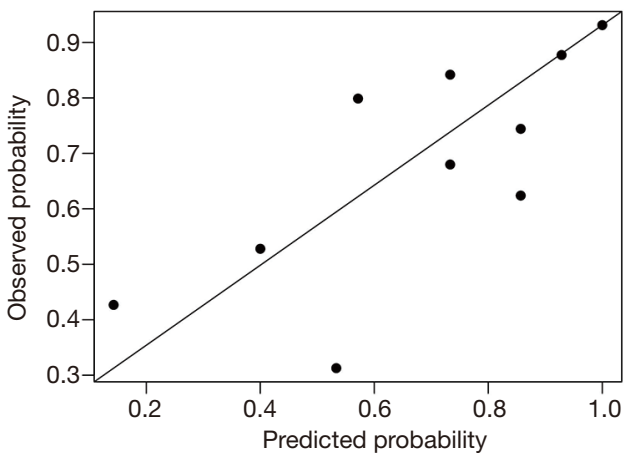

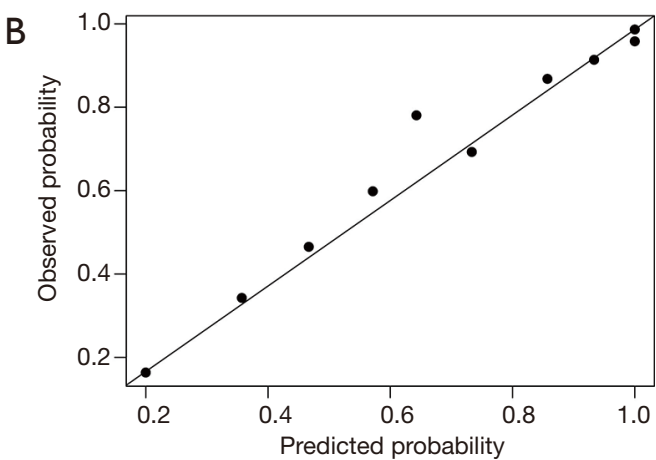

Figure 4 Calibration of the logistic regression models. (A, model 1) molecular subtype and US ( $\Delta$ diameter); (B, model 2) molecular subtype, US ( $\triangle$ diameter) and CEUS ( $\triangle \mathrm{IMAX}, \Delta \mathrm{TTP})$ parameters. CEUS, contrast-enhanced ultrasound; US, ultrasound.

might be superior to a single index in predicting responses to NAC therapy. Calibration of the two models can be seen in Figure 4.

\section{Discussion}

Changes in CEUS parameters after 2 cycles of NAC therapy in breast cancer patients were strongly associated with good therapeutic responses. A combination of CEUS parameters and molecular subtype (model 2) exhibited a good performance (AUC $=0.841$ ) in the prediction of a good response when compared to a combination of the conventional US and molecular subtype (model 1).

Studies have documented that CEUS parameters can be used to predict responses to NAC $(17,21,23,24)$. Lee et al. (21) reported that, in breast cancer, mTT changes were correlated with responses to NAC. In addition, RT has been shown to have a fair predictive value for responses during treatment $(\mathrm{AUC}=0.710)(23)$. However, in this study, mTT and RT were not found to be clinical response predictors to treatment. A possible explanation for the discrepancy between these findings could be attributed to differences in sample sizes and study designs. Previous studies used sample sizes of 17 to 92 cases, while we used a sample size of 143 cases. Other studies tried to established an early prediction window at first cycle of chemotherapy
$(21,23)$. We performed the CEUS examination after two cycles, which was considered a better assessment point to provide accurate and early information (28).

Using changes in diameter, the CEUS model (model 2) with $\triangle \mathrm{IMAX}$ and $\triangle \mathrm{TTP}$ exhibited better performances than the conventional US model (model 1). This could be attributed to the anti-angiogenic effect of chemotherapy that decreases the concentration of vascular growth factor (VGF) within the tumor. The corresponding slowdown kinetics might appear before changes in tumor size (Figure 2B). Furthermore, CEUS enhancement was found in the minor response group and was accompanied by a decrease in US size (Figure 2C), indicating that there was a persistence of small tumor cell nests with regular vascularization. In agreement with previous studies $(17,25)$, our results showed that $\triangle \mathrm{IMAX}, \triangle \mathrm{TTP}$, and $\Delta$ diameter were significant independent predictors of good response to NAC. Compared to previous studies (AUC $=0.710-0.946$ ), our model exhibited a moderate discriminative performance ( $\mathrm{AUC}=0.841$ ). The differences were attributed to different primary endpoints and CEUS parameters in each study. Currently, there is no universally accepted response evaluation criteria for NAC therapy, which may influence trail reporting and interpretation.

Our study confirmed that a combination of pathological and imaging variables is a more accurate predictor of therapeutic 
responses than any of the variables alone $(29,30)$. The molecular subtype was found to be a prime predictor of clinical outcomes in our models. This might contribute to different chemosensitivities among molecular subtypes. Mazari et al. (31) reported that the HER2+ subtype is a significant predictor for pathologically complete responses, and that the odds ratios increased from 2.49 to 5.4 when univariate regression analysis was changed to multivariate regression modeling. In concordance with previous studies $(3,32)$, our results showed that triple-negative [odds ratio (OR): 2.872, 95\% confidence interval (CI): 0.947-16.372] and HER2+ (OR: 3.939, 95\% CI: $0.947-16.372)$ tumors exhibited better responses than hormone receptor-positive, HER2- breast cancer.

This study was limited by: (I) the sample size in this exploratory study was relatively small. In the luminal A subgroup, a relatively small number of patients were enrolled $(20 / 143,13.9 \%)$, and these patients were considered to be less sensitive to and would benefit less from NAC therapy (33). Further studies are required to assess stratified subgroups with larger sample sizes, which could provide sufficient statistical power to address the impact of breast cancer subtype on CEUS parameters. (II) We did not perform an external validation of the fitted model. However, we conducted bootstrap validation to prevent over-interpretation, and the results suggested a satisfactory validation of the model. (III) Utilization of a single CEUS imaging plane to measure the maximum tumor diameter may not represent the entirety of the tumor. Moreover, quantitative results of CEUS are subjective and are depending on the ROIs as well as on the ultrasound company's own program and analysis method.

Nevertheless, our exploratory analyses generate hypotheses for further research. Comprehensive and objective tumor data from different breast cancer subtypes can be incorporated using quantitative high-throughput features referred to as radiomics, which have previously been described (34). If the predictive model for response performs well in a larger group of breast cancer subtypes, it will enable the establishment of an algorithm with cut-off values to predict responses to NAC. There are indications that multimodality imaging, including US (35), mammography, MRI (36) and PET/CT (37), may confer additional values when evaluating responses to NAC. Incorporation of these analyses in our further research is ongoing.

\section{Conclusions}

Parametric changes are associated with clinical responses to NAC. Therefore, clinical responses to NAC can be predicted by a combination of CEUS parameters and molecular subtypes during treatment. Our future study will benefit from a larger patient group and from exploring the correlation between CEUS parameters and clinical relevance. Prognostic factors such as recurrence-free survival and overall survival as well as surgical factors such as second surgical rate will be considered.

\section{Acknowledgments}

We thank the physicians of the Breast Surgery Department and Clinical Statistics Center of Fudan University Shanghai Cancer Center for their contributions to case acquisition and statistical analysis.

Funding: National Natural Science Foundation of China $(81830058,81371575,81801701)$, the Science and Technology Commission of Shanghai Municipality (18411967400, 17411953400), Shanghai Anticancer Association EYAS PROJECT (SACA-CY1C06), Fudan University Shanghai Cancer Center Special Fund for Iconography (YX201804).

\section{Footnote}

Reporting Checklist: The authors have completed the STARD reporting checklist. Available at http://dx.doi.org/10.21037/ gs-20-836

Data Sharing Statement: Available at http://dx.doi. org/10.21037/gs-20-836

Peer Review File: Available at http://dx.doi.org/10.21037/gs20-836

Conflicts of Interest: All authors have completed the ICMJE uniform disclosure form (available at http://dx.doi. org/10.21037/gs-20-836). The authors have no conflicts of interest to declare.

Ethical Statement: The authors are accountable for all aspects of the work in ensuring that questions related to the accuracy or integrity of any part of the work are appropriately investigated and resolved. The study was conducted in accordance with the Declaration of (as revised in 2013). This study had been approved by the Ethical Review Committee of the Fudan University Shanghai Cancer Center (1303119-NSFC) and informed consent was 
taken from all individual participants.

Open Access Statement: This is an Open Access article distributed in accordance with the Creative Commons Attribution-NonCommercial-NoDerivs 4.0 International License (CC BY-NC-ND 4.0), which permits the noncommercial replication and distribution of the article with the strict proviso that no changes or edits are made and the original work is properly cited (including links to both the formal publication through the relevant DOI and the license). See: https://creativecommons.org/licenses/by-nc-nd/4.0/.

\section{References}

1. Gradishar WJ, Anderson BO, Balassanian R, et al. Breast Cancer, Version 4.2017, NCCN Clinical Practice Guidelines in Oncology. J Natl Compr Canc Netw 2018;16:310-20.

2. Masuda N, Lee SJ, Ohtani S, et al. Adjuvant Capecitabine for Breast Cancer after Preoperative Chemotherapy. N Engl J Med 2017;376:2147-59.

3. Cortazar P, Zhang L, Untch M, et al. Pathological complete response and long-term clinical benefit in breast cancer: the CTNeoBC pooled analysis. Lancet 2014;384:164-72.

4. Gounaris I, Provenzano E, Vallier AL, et al. Accuracy of unidimensional and volumetric ultrasound measurements in predicting good pathological response to neoadjuvant chemotherapy in breast cancer patients. Breast Cancer Res Treat 2011;127:459-69.

5. Goorts B, Dreuning KMA, Houwers JB, et al. MRI-based response patterns during neoadjuvant chemotherapy can predict pathological (complete) response in patients with breast cancer. Breast Cancer Res 2018;20:34.

6. Taydaş O, Durhan G, Akpinar MG, et al. Comparison of MRI and US in Tumor Size Evaluation of Breast Cancer Patients Receiving Neoadjuvant Chemotherapy. Eur J Breast Health 2019;15:119-24.

7. Matsuda N, Kida K, Ohde S, et al. Change in sonographic brightness can predict pathological response of triplenegative breast cancer to neoadjuvant chemotherapy. Breast Cancer 2018;25:43-9.

8. Dobruch-Sobczak K, Piotrzkowska-Wroblewska H, Klimonda Z, et al. Ultrasound echogenicity reveals the response of breast cancer to chemotherapy. Clin Imaging 2019;55:41-6.

9. DiCenzo D, Quiaoit K, Fatima K, et al. Quantitative ultrasound radiomics in predicting response to neoadjuvant chemotherapy in patients with locally advanced breast cancer: Results from multi-institutional study. Cancer Med 2020;9:5798-806.

10. Sannachi L, Gangeh M, Tadayyon H, et al. Response monitoring of breast cancer patients receiving neoadjuvant chemotherapy using quantitative ultrasound, texture, and molecular features. PLoS One 2018;13:e0189634.

11. Yuan Y, Failmezger H, Rueda OM, et al. Quantitative image analysis of cellular heterogeneity in breast tumors complements genomic profiling. Sci Transl Med 2012;4:157ra143

12. Choi JH, Lim HI, Lee SK, et al. The role of PET CT to evaluate the response to neoadjuvant chemotherapy in advanced breast cancer: comparison with ultrasonography and magnetic resonance imaging. J Surg Oncol 2010;102:392-7.

13. Izumi Y, Xu L, di Tomaso E, et al. Tumour biology: herceptin acts as an anti-angiogenic cocktail. Nature 2002;416:279-80.

14. Cha JH, Moon WK, Cho N, et al. Differentiation of benign from malignant solid breast masses: conventional US versus spatial compound imaging. Radiology 2005;237:841-6.

15. Rauch GM, Adrada BE, Kuerer HM, et al. Multimodality Imaging for Evaluating Response to Neoadjuvant Chemotherapy in Breast Cancer. AJR Am J Roentgenol 2017;208:290-9.

16. Drudi FM, Cantisani V, Gnecchi M, et al. Contrastenhanced ultrasound examination of the breast: a literature review. Ultraschall Med 2012;33:E1-7.

17. Wan CF, Liu XS, Wang L, et al. Quantitative contrastenhanced ultrasound evaluation of pathological complete response in patients with locally advanced breast cancer receiving neoadjuvant chemotherapy. Eur J Radiol 2018;103:118-23.

18. Saracco A, Aspelin P, Leifland K, et al. Bolus compared with continuous infusion of microbubble contrast agent using real-time contrast harmonic imaging ultrasound in breast tumors. Acta Radiol 2009;50:854-9.

19. Wan CF, Du J, Fang H, et al. Enhancement patterns and parameters of breast cancers at contrast-enhanced US: correlation with prognostic factors. Radiology 2012;262:450-9.

20. Saracco A, Szabo BK, Tanczos E, et al. Contrastenhanced ultrasound (CEUS) in assessing early response among patients with invasive breast cancer undergoing neoadjuvant chemotherapy. Acta Radiol 2017;58:394-402.

21. Lee YJ, Kim SH, Kang BJ, et al. Contrast-Enhanced 
Ultrasound for Early Prediction of Response of Breast Cancer to Neoadjuvant Chemotherapy. Ultraschall Med 2019;40:194-204.

22. Amioka A, Masumoto N, Gouda N, et al. Ability of contrast-enhanced ultrasonography to determine clinical responses of breast cancer to neoadjuvant chemotherapy. Jpn J Clin Oncol 2016;46:303-9.

23. Kim Y, Kim SH, Song BJ, et al. Early Prediction of Response to Neoadjuvant Chemotherapy Using Dynamic Contrast-Enhanced MRI and Ultrasound in Breast Cancer. Korean J Radiol 2018;19:682-91.

24. Zhang Q, Yuan C, Dai W, et al. Evaluating pathologic response of breast cancer to neoadjuvant chemotherapy with computer-extracted features from contrast-enhanced ultrasound videos. Phys Med 2017;39:156-63.

25. Cao X, Xue J, Zhao B. Potential application value of contrast-enhanced ultrasound in neoadjuvant chemotherapy of breast cancer. Ultrasound Med Biol 2012;38:2065-71.

26. Goldhirsch A, Wood WC, Coates AS, et al. Strategies for subtypes--dealing with the diversity of breast cancer: highlights of the St. Gallen International Expert Consensus on the Primary Therapy of Early Breast Cancer 2011. Ann Oncol 2011;22:1736-47.

27. Ogston KN, Miller ID, Payne S, et al. A new histological grading system to assess response of breast cancers to primary chemotherapy: prognostic significance and survival. Breast 2003;12:320-7.

28. Avril N, Sassen S, Roylance R. Response to therapy in breast cancer. J Nucl Med 2009;50 Suppl 1:55S-63S.

29. Antunovic L, De Sanctis R, Cozzi L, et al. PET/CT radiomics in breast cancer: promising tool for prediction of pathological response to neoadjuvant chemotherapy. Eur J Nucl Med Mol Imaging 2019;46:1468-77.

30. Pengel KE, Koolen BB, Loo CE, et al. Combined use of (1)(8)F-FDG PET/CT and MRI for response monitoring of breast cancer during neoadjuvant chemotherapy. Eur J

Cite this article as: Huang $\mathrm{Y}$, Le J, Miao A, Zhi W, Wang F, Chen Y, Zhou S, Chang C. Prediction of treatment responses to neoadjuvant chemotherapy in breast cancer using contrastenhanced ultrasound. Gland Surg 2021;10(4):1280-1290. doi: 10.21037 /gs-20-836
Nucl Med Mol Imaging 2014;41:1515-24.

31. Mazari FAK, Sharma N, Dodwell D, et al. Human Epidermal Growth Factor 2-positive Breast Cancer with Mammographic Microcalcification: Relationship to Pathologic Complete Response after Neoadjuvant Chemotherapy. Radiology 2018;288:366-74.

32. Boughey JC, McCall LM, Ballman KV, et al. Tumor biology correlates with rates of breast-conserving surgery and pathologic complete response after neoadjuvant chemotherapy for breast cancer: findings from the ACOSOG Z1071 (Alliance) Prospective Multicenter Clinical Trial. Ann Surg 2014;260:608-14; discussion 14-6.

33. Nielsen TO, Jensen MB, Burugu S, et al. High-Risk Premenopausal Luminal A Breast Cancer Patients Derive no Benefit from Adjuvant Cyclophosphamide-based Chemotherapy: Results from the DBCG77B Clinical Trial. Clin Cancer Res 2017;23:946-53.

34. Hu Y, Qiao M, Guo Y, et al. Reproducibility of quantitative high-throughput BI-RADS features extracted from ultrasound images of breast cancer. Med Phys 2017;44:3676-85.

35. Zhang K, Li J, Zhu Q, et al. Prediction of Pathologic Complete Response by Ultrasonography and Magnetic Resonance Imaging After Neoadjuvant Chemotherapy in Patients with Breast Cancer. Cancer Manag Res 2020;12:2603-12.

36. Gampenrieder SP, Peer A, Weismann C, et al. Radiologic complete response (rCR) in contrast-enhanced magnetic resonance imaging (CE-MRI) after neoadjuvant chemotherapy for early breast cancer predicts recurrencefree survival but not pathologic complete response (pCR). Breast Cancer Res 2019;21:19.

37. Humbert O, Lasserre M, Bertaut A, et al. Breast Cancer Blood Flow and Metabolism on Dual-Acquisition (18) F-FDG PET: Correlation with Tumor Phenotype and Neoadjuvant Chemotherapy Response. J Nucl Med 2018;59:1035-41. 